\title{
GROUPS WITH CENTRAL 2-SYLOW INTERSECTIONS OF RANK AT MOST ONE
}

\author{
MARCEL HERZOG AND ERNEST SHULT
}

\begin{abstract}
An involution in a finite group is called central if it lies in the center of a 2-Sylow subgroup of $G$. A 2-Sylow intersection is called central if it is either trivial or contains a central involution. Suppose $G$ is a finite simple group all of whose central 2-Sylow intersections are trivial or rank one 2-groups. It is proved that $G$ is a known simple group.
\end{abstract}

1. Introduction. All groups considered in this paper are finite. An involution is called central if it lies in the center of some 2-Sylow subgroup of $G$. An intersection of distinct 2-Sylow groups is called a central 2Sylow intersection if it is trivial or contains a central involution.

The objective of this paper is to prove the following.

THEOREM. Let $G$ be a simple group. Suppose all central 2-Sylow intersections of $G$ have 2-rank at most one. Then $G$ is isomorphic to one of the following groups:

(i) $\operatorname{PSL}(2, q), q=2^{n}>2$,

(ii) $S z(q), q=2^{n} \geqq 8$,

(iii) $\operatorname{PSU}(3, q), q=2^{n}>2$,

(iv) $\operatorname{PSL}(2, q), q \equiv 3$ or $5(\bmod 8), q>5$,

(v) $J$, the Janko group of order 175,560.

In this paper, the groups of type (i), (ii) and (iii) are called "simple Bender groups". Throughout, $S(G)$ denotes the largest solvable normal subgroup of $G$. The rest of the notation used is standard.

2. Previous results. Of basic importance is

THEOREM 2.1. Let $z$ be a central involution in G. Suppose $z$ lies in no proper 2-Sylow intersection of $G$. Then $\left\langle z^{G}\right\rangle$, the normal closure of $z$ in $G$, has a center of odd order and, modulo this center, is the direct product of simple Bender groups and a 2-nilpotent group having 2-Sylow subgroup of exponent 2. $z$ projects nontrivially on each of the components of this factor.

Received by the editors April 17, 1972 and, in revised form, June 9, 1972.

AMS (MOS) subject classifications (1969). Primary 2025; Secondary 2027, 2029. Key words and phrases. Finite simple group, 2-Sylow intersection.

(c) American Mathematical Society 1973 
This is basically the theorem in [3], where it is proved that $z$ satisfies the hypotheses of the fusion theorem of [8] (see also [2, p. 62], for a statement of the fusion theorem).

THEOREM 2.2. Let $G$ be a simple group with all central 2-Sylow intersections having rank at most one. Suppose for some central involution $z$ in $G$, $C_{G}(z)$ is solvable. Then $G$ is isomorphic to either $S L\left(2,2^{a}\right), S z\left(2^{n}\right)$ (nodd), $U\left(3,2^{a}\right)$, or $\operatorname{PSL}(2, q), q \equiv 3$ or $5(\bmod 8)$.

This is the main theorem of [4].

THEOREM 2.3. Let $G$ be a simple group with all central 2-Sylow intersections cyclic. Then $G$ is isomorphic to $S L\left(2,2^{a}\right), S z\left(2^{n}\right)(n$ odd $), U\left(3,2^{a}\right)$, $\operatorname{PSL}(2, q), q \equiv 3$ or $5(\bmod 8)$ or J, Janko's group of order 175,560.

This is the principal theorem of [5]. It generalizes a theorem of Mazurov [6] which characterizes simple groups with all proper 2-Sylow intersections cyclic.

REMARK. The proof of the theorem of the introduction appears in the next section, and utilizes all of the results just quoted. There exists a somewhat longer proof which proceeds from "first principles" in the sense that dependence on [4], [5] and Mazurov's theorem is avoided. This proof requires, however, a "weak closure" characterization of Janko's group [9].

It should be remarked that Mazurov's theorem has also been generalized in the direction of considering groups, all of whose proper 2-Sylow intersections have rank at most one. The classification of groups bearing this hypothesis was first accomplished by Michael Aschbacher [0], and independently by Peter Landrock [7]. In both of these papers $G$ is not assumed to be simple.

3. Proof of the theorem. Let $G$ be a counterexample, that is, $G$ satisfies Hypothesis 3.1.

(a) $G$ is a simple group;

(b) all central 2-Sylow intersections of $G$ have rank at most one;

(c) $G$ is not isomorphic to one of the groups $S L\left(2,2^{a}\right), S z\left(2^{n}\right)$ (n odd), $U\left(3,2^{a}\right), \operatorname{PSL}(2, q), q \equiv 3$ or $5(\bmod 8)$ or $J$.

The proof proceeds by a series of short steps showing that Hypothesis 3.1 is inconsistent.

(i) The centralizers of all central involutions of $G$ are nonsolvable.

This follows at once from Theorem 2.2.

(ii) $G$ possesses a quaternion central 2-Sylow intersection.

Otherwise, by Theorem 2.3, $G$ must be a group forbidden by Hypothesis 3. 1 (c). 
(iii) Define the following sets:

$\mathscr{M}=\left\{N_{G}(V) \mid V\right.$ is a 2-subgroup of $G$ containing a central involution. $N_{G}(V)$ is nonsolvable and contains a 2-Sylow subgroup of $\left.G\right\}$.

$\mathscr{N}=\left\{H|H \in \mathscr{M},| O_{2}(H)|\geqq| O_{2}\left(H_{1}\right) \mid\right.$ for all $\left.H_{1} \in \mathscr{M}\right\}$, the elements of $\mathscr{M}$ with $\mathrm{O}_{2}$ as large as possible.

$\mathscr{N}^{*}=\{$ the maximal elements of $\mathscr{N}\}$.

$\mathscr{N}^{*}$ is not empty.

It suffices to show $\mathscr{M}$ is nonempty. But by (i), if $z$ is any central involution, $C_{G i}(z) \in \mathscr{M}$.

(iv) Let $H$ be a fixed element of $\mathscr{N}^{*}$. All proper 2-Sylow intersections of $H$ are central 2-Sylow intersections of $G$ and so are rank one.

Since $H$ lies in $\mathscr{N}^{*},|H|_{2}=|G|_{2}$ and $H=N_{G}\left(V_{0}\right)$ where $V_{0}$ is a nontrivial 2-group containing a central involution of $G$. Thus all proper 2-Sylow intersections of $H$ are central 2-Sylow intersections of $G$.

(v) Set $S=S(H)$, the largest normal solvable subgroup of $H$. Let $V$ be a 2-Sylow subgroup of $S$. Then $H=N_{G}(V)$ and $V=O_{2}(H)$ is a rank one 2group.

By a Frattini argument, $H=S N_{H}(V)$ and so $N_{G}(V)$ is nonsolvable and $\left|N_{G}(V)\right|_{2}=\left|N_{H}(V)\right|_{2}=|H|_{2}=|G|_{2}$. Thus $N_{G}(V)$ lies in $\mathscr{M}$. Now

$$
O_{2}\left(N_{G}(V)\right) \geqq V \geqq O_{2}(S(H))=O_{2}(H) .
$$

Since $H$ lies in $\mathscr{N}, O_{2}\left(N_{G}(V)\right)=V=O_{2}(H)$ and this forces $H=N_{G}(V)$, $V=O_{2}(H)$. Since $H$ (being nonsolvable) is not 2-closed, $V$ lies in a proper 2-Sylow intersection of $H$ and this intersection is rank one by (iv). Hence $V$ has rank one.

(vi) Let $z$ be the unique involution in $V$. Then for any element $x$ of $G$, $z^{x}=x^{-1} z x$ lies in the center of every 2-Sylow subgroup of $G$ which contains it.

Some conjugate $z^{g}$ lies in $H-\{z\}$. In the group $\bar{H}=H / S, \bar{z}^{g}$ is a central involution which does not belong to any proper 2-Sylow intersection of $\bar{H}$.

Suppose $P$ is a 2-Sylow subgroup of $G$ containing $z$ in its center. Suppose $z^{x}$ lies in $a^{-1} P a$. Then if $z^{x}$ were not in the center of $a^{-1} P a,\left\langle z^{x}, z^{a}\right\rangle$ would be a fours-group lying in the intersection $a^{-1} P a \cap(x c)^{-1} P(x c)$ where $c$ is an appropriately chosen element of $C_{G}\left(z^{x}\right)$. Hypothesis $3.1(\mathrm{~b})$ forces $a^{-1} P a=$ $c^{-1} x^{-1} P x c$, so $a^{-1} x c \in N_{G}\left(a^{-1} P a\right)$. But $z^{a}$ and $z^{x}=z^{x c}$ are conjugate under the action of $a^{-1} x c$. Thus $z^{x}$ must also lie in the center of $a^{-1} P a$ contrary to assumption. Thus $z^{x} \in Z\left(a^{-1} P a\right)$ and the first assertion is proved.

Now let $P$ be a 2-Sylow subgroup of $H$. Then, since $G$ is simple (Hypothesis 3.1(a)), Glauberman's $Z^{*}$-theorem [1] requires the existence of a conjugate $z^{g}$ in $P-\{z\}$. Then since $z$ is the unique involution in $P \cap S=V$ (by (v)), $z^{g}$ does not lie in $S$. Since $z^{g}$ lies in $Z(P)$ by the first part of (vi), we see that $\bar{z}^{g}$ is a central involution of $\bar{H}$. Now suppose $\bar{z}^{g}$ could be 
realized as an element in a proper 2-Sylow intersection $\bar{R}_{1} \cap \bar{R}_{2}$ of $\bar{H}$. Then there exist 2-Sylow subgroups $R_{1}$ and $R_{2}$ of $H$, each containing the element $z^{g}$, such that $R_{i} S / S=\bar{R}_{i}$. Then since $R_{i} \cap S=V, R_{1} \cap R_{2}$ contains the fours-group $\left\langle z^{g}, z\right\rangle$ contrary to Hypothesis 3.1(b). Thus $\bar{z}^{g}$ cannot lie in a proper 2-Sylow intersection of $\bar{H}$.

(vii) Let $\bar{L}$ be the normal closure of $\bar{z}^{g}$ in $\bar{H}$. Then $\bar{L}$ is a direct product of Bender groups.

Because of (vi) we may apply Theorem 2.1 to $\bar{H}$ and the involution $\bar{z}^{g}$. Since $H / S$ has no nontrivial normal solvable subgroups, $S(\tilde{L})=1$ and so $\bar{L}$ is semisimple.

(viii) $\bar{L}$ is a simple Bender group.

Since a 2-Sylow intersection in $\bar{H}$ can be realized as the homomorphic image of a 2-Sylow intersection of $H$, by (iv), such intersections in $\bar{H}$ have rank at most 2 . Suppose $L$ is not a simple Bender group. Then we must have $L \simeq L_{1} \times L_{2}$ where $L_{i} \simeq S L(2,4)$ or $U(3,4)$. Let $\bar{T}$ be a 2-Sylow center of $L$ and choose $T$ as 2-Sylow subgroup of the preimage of $\bar{T}$.

Let $\bar{T}_{i}$ denote the 2-Sylow center of $L_{i}, i=1,2$, and let $T_{i}$ be the preimage of $\bar{T}_{i}$ in the homomorphism $T \rightarrow T S / S=\bar{T}$. Then $T=T_{1} T_{2}, T_{1} \cap T_{2}=$ $T \cap S=V$. Each $T_{i}$ lies in a proper 2-Sylow intersection of $H$, and has sectional 2-rank at least 2. Thus each $T_{i}$ is generalized quaternion. Thus $Z(T)=Z\left(T_{1} T_{2}\right) \leqq T \cap S=V$. But this is impossible since $T$ is generated by $V$ and conjugates of $z^{g}$ which necessarily lie in $Z(T)-V$ by (vi).

(ix) $\bar{H} \mid \bar{L}$ is 2-closed. $\bar{L}$ is the unique minimal normal subgroup of $\bar{H}$. $C_{H}(\bar{L})=1$.

Let $T$ be a 2-Sylow subgroup of the preimage $T$ of a 2-Sylow center of $\bar{L}$ in $H$, containing a conjugate $z^{g}$ of $z$, outside of $T \cap S=V$. Then by (vi), $\left\langle z^{g}, z\right\rangle$ is a fours-group and so by Hypothesis 3.1(b), $N_{G}(T)$ is 2-closed. But if $L$ is the preimage of $\bar{L}$ in $H, N_{H}(T) L=H$ so $\bar{H} \mid \bar{L}$ is a homomorphic image of $N_{H}(T)$ and is therefore 2-closed.

Let $\bar{K}$ be a minimal normal subgroup of $\bar{H}$ distinct from $\bar{L}$. Then $\bar{K} \cap \bar{L}=$ 1 and so $R$ is isomorphic to a subgroup of $\bar{H} \mid \bar{L}$ and is therefore 2-closed. But then $R \leqq S(\bar{H})=1$, a contradiction.

Since $C_{H}(\bar{L})$ is a normal subgroup of $\bar{H}$ meeting $\bar{L}$ trivially, it must also be trivial, since $L$ is the unique minimal normal subgroup of $\bar{H}$.

(x) $\bar{H} / \bar{L}$ has odd order.

As before let $L$ denote the preimage of $L$ in $H$ and let $T$ be the 2-Sylow subgroup of the preimage of a 2-Sylow center of $\bar{L}$. Then as we have seen $N_{G}(T)$ is 2-closed. Let $P$ be a 2-Sylow subgroup of $H$ containing $T$. Then $T$ is normal in $P$. The conjugates of $z$ lying in $T-V$ span $T / V$ and are central in $P$. Thus conjugation by elements in $P$ induces a 2-group of automorphisms of $\bar{L}$ each of which:

(a) centralizes a 2-Sylow center $\bar{T}$ of $\bar{L}$, and 
(b) centralizes $N_{L}(\bar{T}) /(\bar{P} \cap \bar{L})$, a cyclic group of order $q^{\delta}-1, q=|\bar{T}|$, $\delta=1,1$, or 2 .

Condition (b) occurs because $\bar{P} \leqq N_{H}(\bar{T})$ and the latter is 2-closed (being a section covered by $N_{G}(T)$ ). All automorphisms of the Bender groups satisfying conditions (a) and (b) are inner. Thus we obtain the factorization

$$
\bar{P}=C_{P}(\bar{L}) \times(\bar{P} \cap \bar{L}) .
$$

By (ix) the first factor is trivial. Thus $\bar{P} \leqq L$ and so (x) holds.

(xi) $V$ has order 2.

$V$ is a rank 12 -group with unique involution $z$. Let $P$ be a 2-Sylow subgroup of $H$. By (x), $P S / S=\bar{P}$ is a 2-Sylow subgroup of the Bender group $\bar{L}$. There exists a conjugate $z^{g}$ in $P-V$. Since $z$ and $z^{g}$ both lie in the center of $P$ we may assume without loss of generality that $g \in N_{G}(P)$. Then $V^{g}$ is a normal subgroup of $P$ with unique involution $z^{g}$. Thus $V^{g} \cap V=1$ and $V^{g} S / S=\bar{V}^{g}$ is a rank 1 normal subgroup of a 2-Sylow subgroup $\bar{P}$ of a Bender group. But the only such normal subgroups of a 2-Sylow subgroup of a Bender group have order 2. Hence $\left|V^{g}\right|=2$. This implies (xi).

(xii) Let $P$ be a 2-Sylow subgroup of $H$. Then by (x), $\bar{P}=P S / S$ is a 2Sylow subgroup of the Bender group $\bar{L}$. Let $\bar{T}$ denote the center of $\bar{P}$ and let $T$ be the preimage of $\bar{T}$ in $P$. Then $T$ is elementary, $T=\Omega_{1}(P), T=Z(P)$ and $T^{\#}$ is fused in $N_{G}(P)$.

Clearly $\left\langle z^{G} \cap P\right\rangle \leqq T$ since $\bar{T}=\Omega_{1}(\bar{P})$. But since $N_{L}(\bar{P})$ transitively conjugates the $q-1$ elements of $\bar{T}$ and some $z^{g}$ lies in $T-V$ and $V=\langle z\rangle$, we have

$$
T=\left\langle z^{G} \cap P\right\rangle .
$$

Then $T \leqq Z(P)$. Since $\bar{T}=Z(\bar{P}), T=Z(P)$. Since $T$ is generated by mutually commuting involutions it is elementary, hence $T \leqq \Omega_{1}(P)$. But $\bar{T}=\Omega_{1}(\bar{P})$ so $T=\Omega_{1}(P)$. Now $T$ is elementary of order $2 q$ and its nonzero elements are permuted by a subgroup of $L$ into three orbits of lengths $1, q-1$, and $q-1$. Here $\{z\}$ comprises the orbit of length one, and one of the other orbits contains $z^{g}$. But since $z^{G} \cap P$ (being a subset of the center of $P$ ) is fused in $N_{G}(P)$ we see that $\left|z^{G} \cap P\right|$ is odd and so $z^{G} \cap P=T^{\#}$ This set is fused in $N_{G}(P)$.

(xiii) Contradiction.

Suppose $T \neq P$. Then $\bar{L} \simeq U(3, q)$ or $S z(q)$. Suppose $z=w^{2}$ for some $w \in P$. Then $\bar{w}=w S / S$ is an involution in $\bar{P}$ and so lies in $\Omega_{1}(\bar{P})=Z(\bar{P})=\bar{T}$. But then $w \in T$ and so $w^{2}=1$ by (xii), a contradiction. Thus $z$ is not a square in $P$. Then since $T^{\#}$ is fused in $N_{G}(P)$, no element of $T$ is a square. But this is clearly impossible since any element of $P-T$ has order 4 .

Thus $T=P$. Now $P$ is abelian. Since $P$ is a 2-Sylow subgroup of $G$ this contradicts (ii). 


\section{REFERENCES}

0. M. Aschbacher, A class of generalized TI groups, Illinois J. Math. 16 (1972), 529532.

1. G. Glauberman, Central elements in core-free groups, J. Algebra 4 (1966), 403-420. MR 34 \#2681.

2. - Global and local properties of finite groups. Finite simple groups, Academic Press, New York, 1971.

3. M. Herzog, On 2-Sylow intersections, Israel J. Math. 11 (1972), 326-327.

4. - Central 2-Sylow intersections (to appear).

5. - Simple groups with cyclic central 2-Sylow intersections (to appear).

6. V. D. Mazurov, Finite simple group with cyclic intersections of Sylow 2-subgroups, Algebra i Logika 10 (1971), 188-198. (Russian)

7. P. Landrock, Finite groups with Sylow 2-intersections of rank $\leqq 1$, Aarhus University Preprint Series, no. 23 (to appear).

8. E. Shult, On the fusion of an involution in its centralizer (to appear).

9. - - , A note of Janko's simple group of order 175,560, Proc. Amer. Math. Soc. 35 (1972), 342-348.

Department of Mathematics, Aarhus University, Aarhus, Denmark

Department of Mathematics, Tel Aviv University, Tel Aviv, Israel (Current address of M. Herzog)

Department of Mathematics, University of Florida, Gainesville, Florida 32306 (Current address of Ernest Shult) 